\title{
Heavy flavours
}

\section{André Mischke*}

Institute for Subatomic Physics, Department for Physics and Astronomy and EMME $\phi$, Faculty of Science, Utrecht University, Princetonplein 5, 3584 CS Utrecht, the Netherlands

E-mail: a.mischke@uu.nl

The XXII. International Workshop on Deep-Inelastic Scattering and Related Subjects (DIS 2014) was held in April 2014 in Warsaw, Poland. This contribution summarises some of the highlights on Heavy Flavours presented at this conference, focussing on recent results on 'Charm and beauty production', 'Heavy quarks as a probe of hot and dense strongly interacting matter', 'Rare decays, Mixing and New Physics searches' and 'Spectroscopy and Quarkonia'.

XXII. International Workshop on Deep-Inelastic Scattering and Related Subjects, 28 April - 2 May 2014

Warsaw, Poland

${ }^{*}$ Speaker. 

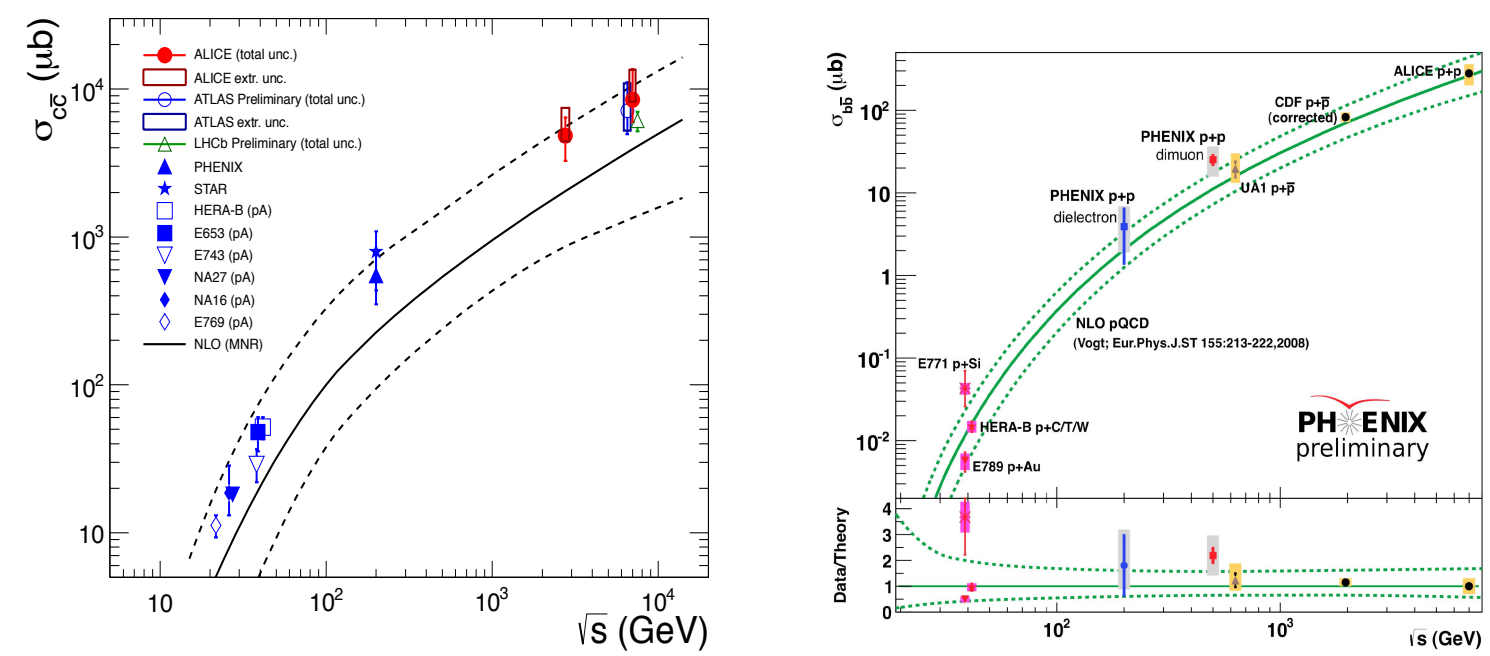

Figure 1: Total nucleon-nucleon charm (left panel) and beauty production cross section (right panel) versus collision energy [1, 2]. The NLO MNR calculation [3] (and its uncertainties) is shown by solid (dashed) lines.

\section{Introduction}

A wealth of new and high precision data from the accelerator facilities DESY-HERA, BNL-RHIC, Tevatron, CERN-LHC and the B-factories have been shown as well as recent developments in theory. This overview contribution will mention briefly a few highlights on heavy flavour at this conference and the reader is referred to the individual theoretical and experimental contributions in these proceedings for details. Only a selection of results could be shown but the content is balance with the one for the summary talk.

\section{Charm and beauty production}

The differential and total charm and beauty production cross section is determined from measurements of open charm and beauty mesons and semi-leptonic decays of heavy flavour hadrons at different centre-of-mass energies. A compilation of the world data on the total charm (beauty) production cross section is depicted in the left (right) panel of Fig. 1, together with next-to-leadingorder (NLO) perturbative QCD calculations [3]. A very good agreement between the different experiments at a certain collision energy is observed. The charm data are systematically higher than the central value of the NLO pQCD calculations, but consistent within theoretical uncertainties. The measured total beauty cross sections are very well described by theoretical model calculations. A combined measurement on the $\mathrm{D}^{\star}$ differential production cross-section was presented by the H1 and ZEUS experiments and is illustrated in Fig. 2. As can been seen a high precision in the measurement was achieved with uncertainties on the data to be much smaller than the uncertainties from the calculation. The NLO pQCD calculation gives a reasonable good description of the data. However, NNLO calculations and an improved fragmentation are needed. 

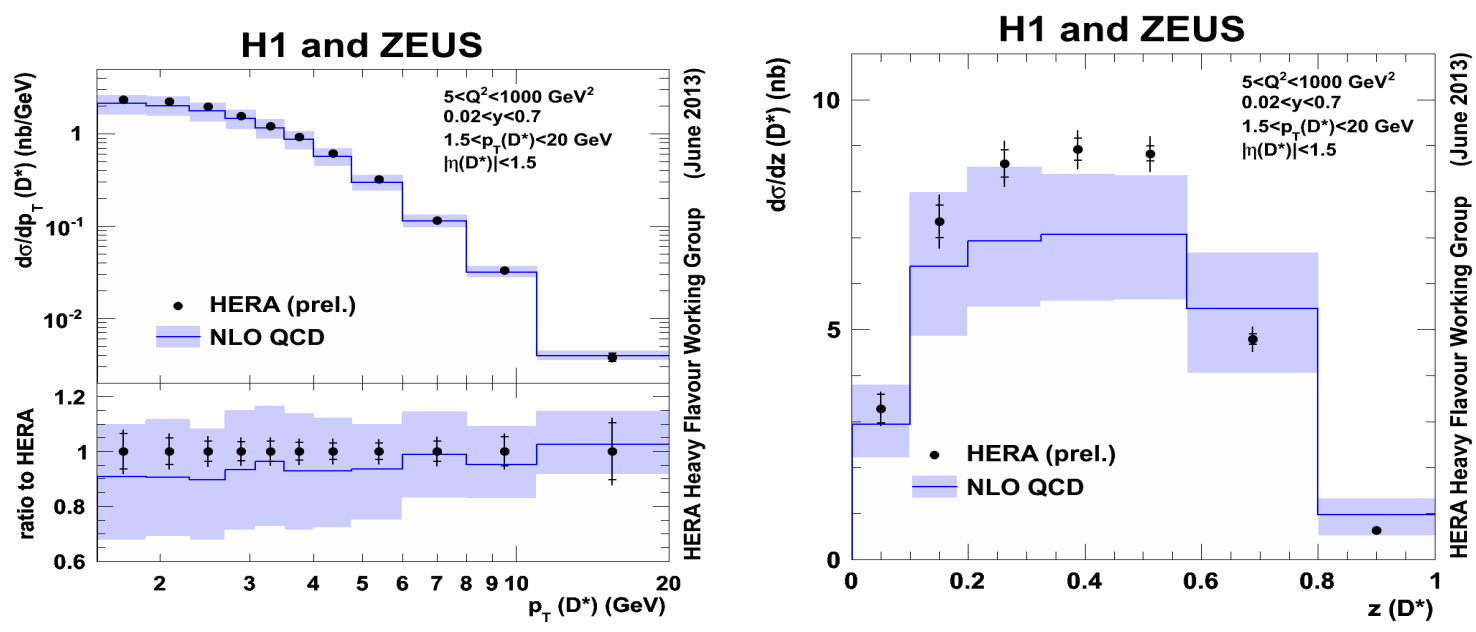

Figure 2: $\mathrm{D}^{\star}$ differential production cross-section at mid-pseudorapidity versus transverse momentum $p_{\mathrm{T}}$ and $z$ for a $Q^{2}$ range $5<Q^{2}<1000 \mathrm{GeV}^{2}$, measured by the $\mathrm{H} 1$ and ZEUS experiments [4]. The purple curves illustrate the central value and the uncertainties of the NLO pQCD calculations.

The ZEUS experiment at HERA provided first measurement on the $\sqrt{s}$-dependence of charm production using $\mathrm{D}^{\star}$ mesons, as illustrated in Fig. 3. It was found that the data, which are sensitive to the gluon distribution, are well described by next-to-leading-order QCD calculations. The data provided confidence in the predictions for higher $\sqrt{s}$, e.g. for the expected energy regime of the LHeC facilitiy.

As illustrated in Fig. 4, left panel, the relative yield for $\mathrm{D}^{0}, \mathrm{D}^{+}$and $\mathrm{D}^{*+}$ mesons in the $p_{\mathrm{T}}$ range 2-4 GeV/c increase linearly with the charged particle density. Inclusive $J / \psi$ follows this trend. The

\section{ZEUS}

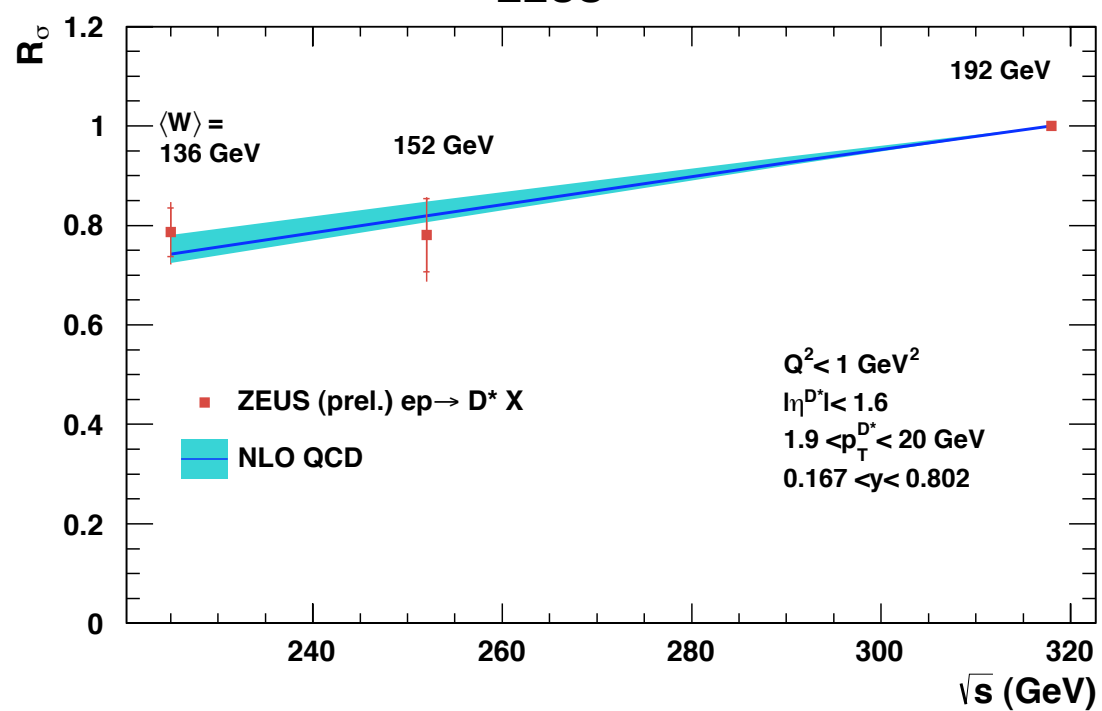

Figure 3: $\sqrt{s}$-dependence of $\mathrm{D}^{\star}$ meson production at mid-rapidity in $e p$ collisions measured by the ZEUS experiment at HERA [5]. The blue curve shows NLO pQCD calculations, including the uncertainty band. 

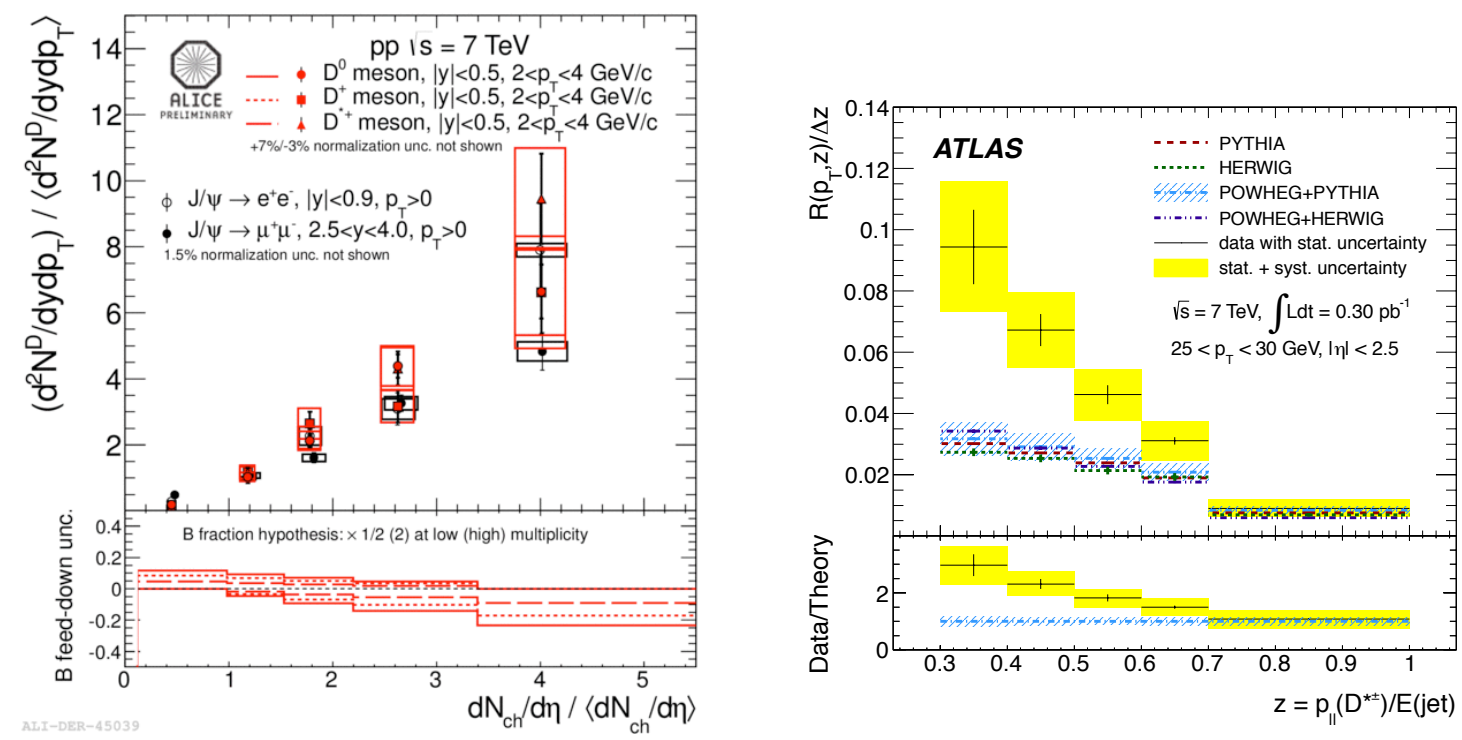

Figure 4: Left: Relative yield for $\mathrm{D}^{0}, \mathrm{D}^{+}$and $\mathrm{D}^{*+}$ for $2<p_{\mathrm{T}}<4 \mathrm{GeV} / c$ and inclusive $J / \psi$ versus charged particles multiplicity $[6,14]$. Right: $\mathrm{D}$ production rate $R\left(p_{\mathrm{T}}, z\right) / \Delta z$ in the jet $p_{\mathrm{T}}$ range $25-30 \mathrm{GeV}$ versus $\mathrm{z}$, measured in $7 \mathrm{TeV}$ pp interactions by ATLAS [7]. The data are compared with the predictions of the Monte Carlo event generators PYTHIA, HERWIG, POWHEG+PYTHIA and POWHEG+HERWIG. The insets show the ratio of the measurement to the POWHEG+PYTHIA prediction.

data might be interpreted in terms of multi-parton interaction in the initial stage of the collision [8].

Moreover, the ATLAS experiment has measured the $\mathrm{D}^{*+}$ meson production in jets in proton-proton collisions at $\sqrt{s}=7 \mathrm{TeV}$ for jets with transverse momentum between 25 and $70 \mathrm{GeV}$ in the pseudorapidity range $|\eta|<2.5$ [7]. Figure 4 (right panel) depicts the $\mathrm{D}^{*+}$ jet production rate $(R)$ for jets in the $p_{\mathrm{T}}$ range $25-30 \mathrm{GeV} / c$ and $|\eta|<2.5$. The data are compared to state-of-the-art NLO pQCD calculations using the Monte Carlo event generators PYTHIA and HERWIG for parton showering. The predicted values of production rate by PYTHIA and POWHEG+PYTHIA are very similar, which is also the case when comparing calculations from HERWIG and POWHEG+HERWIG, as expected. Since $R$ is defined as the ratio between the number of $\mathrm{D}^{*+}$ jets and inclusive jets, the changes of total jet cross sections and $p_{\mathrm{T}}$ distributions between LO and NLO QCD calculations largely cancel. However, the Monte Carlo calculations fail to describe the data at small fractional momentum of the $\mathrm{D}^{*+}$ mesons (z), and this discrepancy is strongest at low jet transverse momentum. The differences observed between the data and model predictions cannot be explained by varying the mixture of charm and beauty jets in the Monte Carlo calculations [7]. This is an indication that jet fragmentation into $\mathrm{D}^{*+}$ mesons is not well modelled in current Monte Carlo generators.

Figure 5 shows the cross section for double charm (D-D meson pairs) production in $p p$ collisions at $\sqrt{s}=7 \mathrm{TeV}[9,10,11]$. Single and double parton scattering become comparable at $\mathrm{TeV}$ energies. The theoretical model calculations are compared with data from the LHCb experiment and shows reasonable good agreement. Contributions from double- $J / \psi$ production are not considered yet and need to be implemented in the model calculations. 

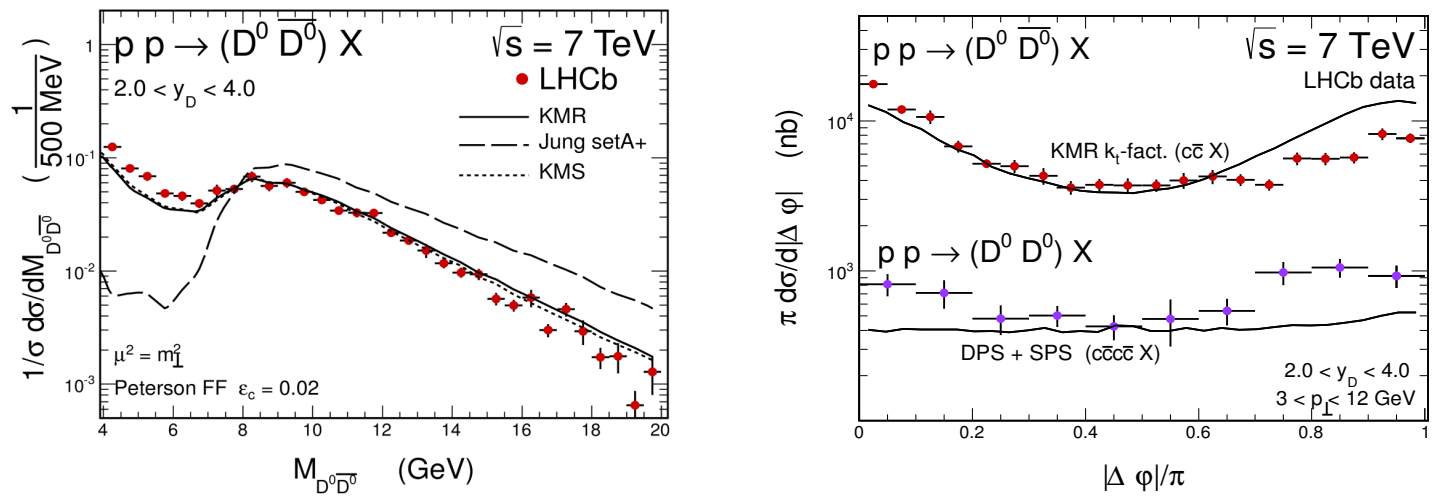

Figure 5: Cross section for double charm (D-D meson pairs) production in $p p$ collisions at $\sqrt{s}=7 \mathrm{TeV}[9$, 10, 11], compared with data from the LHCb experiment.

\section{Heavy quarks as a probe of hot and dense strongly interacting matter}

High energy collisions of heavy atomic nuclei allow to create and carefully study a high-density, colour-deconfined state of strongly-interacting matter. According to calculations from lattice Quantum-Chromodynamics, under the conditions of high energy density and temperature reached in such collisions, the phase transition to a quark-gluon plasma (QGP) is expected to occur, where the colour confinement of quarks and gluons into hadrons should vanish and chiral symmetry should be restored. Heavy-flavour particles, containing charm and beauty, are ideal probes of the conditions of the medium formed in nucleus-nucleus collisions at high energy since they experience the full space-time evolution of the expanding system. They travel through the medium and lose energy due to gluon radiation and multiple collisions. Beauty quarks are even three to four times heavier than charm quarks (but much less abundant).

Of particular interest is the dependence of the parton energy loss on the colour charge and quark mass, which in term allows to gain more information about the transport properties of the QCD matter. Due to their large mass $\left(>1.3 \mathrm{GeV} / \mathrm{c}^{2}\right)$, heavy quarks (charm and beauty) are formed predominantly in the earlier stage of the collision by initial hard scatterings through gluon fusion processes. Charm quarks are about 250 times heavier than the light up and down quarks that dominate the QGP matter and their mass is not affected by chiral symmetry breaking [?]. Therefore, they allow to test QCD at transition from perturbative to non-perturbative regime. The formation time $t_{\mathrm{f}}$ for charm quarks is around $0.1 \mathrm{fm} / c$ and $0.02 \mathrm{fm} / c$ for beauty quarks $\left(t_{\mathrm{f}} \simeq 1 / 2 m_{\mathrm{q}}\right)$.

Nuclear effects are typically quantified using the nuclear modification factor $R_{\mathrm{AA}}$ where the particle yield in nucleus-nucleus collisions is divided by the yield in pp reactions scaled by the number of binary collisions. $R_{\mathrm{AA}}=1$ would indicate that no nuclear effects, such as Cronin effect, shadowing or gluon saturation, are present and that nucleus-nucleus collisions can be considered as an incoherent superposition of nucleon-nucleon interactions.

The left panel of Fig. 6 shows the transverse momentum dependence of the nuclear modification factor $R_{\mathrm{AA}}$ for $\mathrm{D}^{0}$ mesons in the $\mathrm{Au}+\mathrm{Au}$ collisions at $\sqrt{s_{\mathrm{NN}}}=200 \mathrm{GeV}$ from the STAR experiment at the Relativistic Heavy Ion Collider [12]. The $R_{\mathrm{AA}}$ is enhanced at around $1.7 \mathrm{GeV} / c$ and shows a 

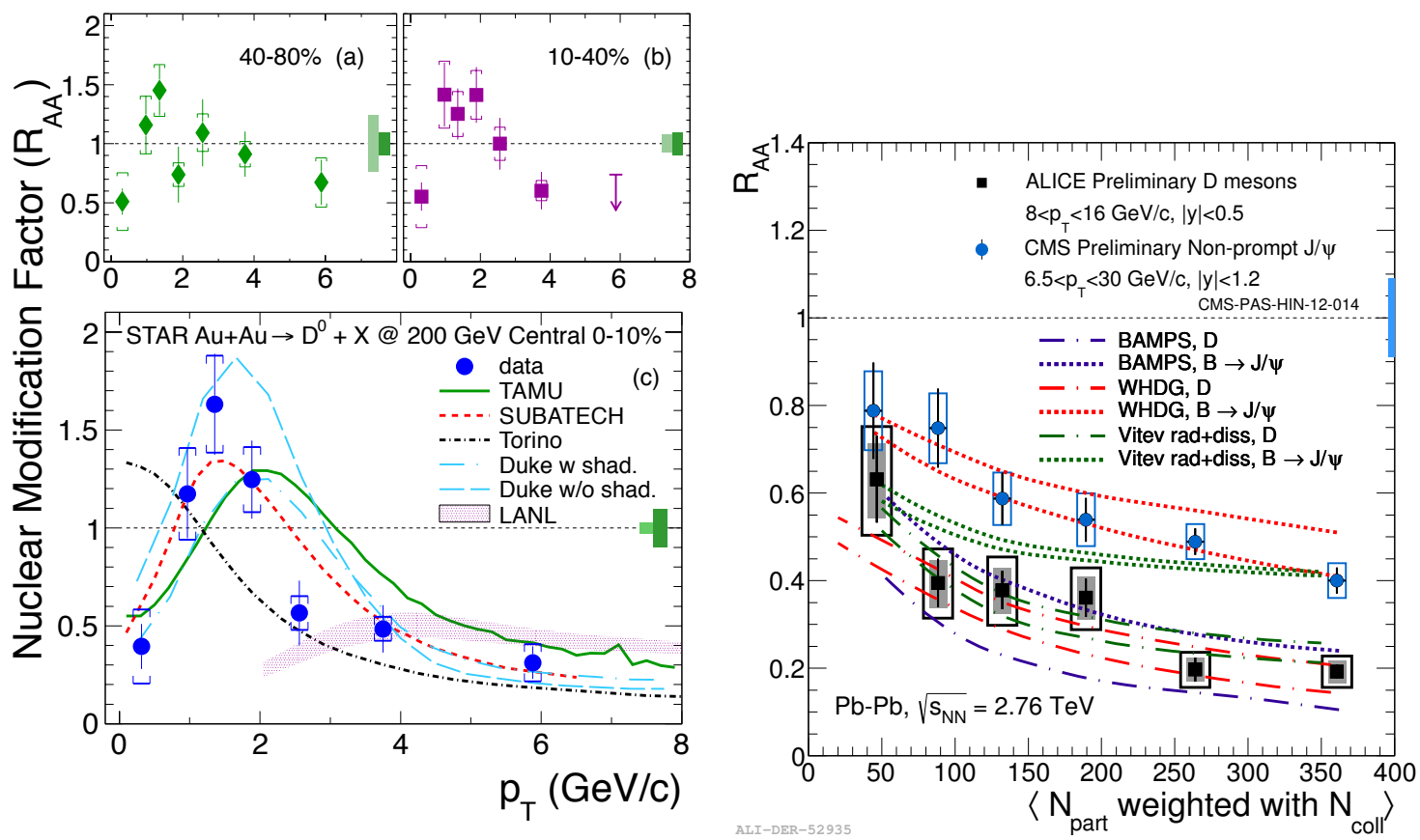

Figure 6: Left panel: Transverse momentum dependence of the average $R_{\mathrm{AA}}$ of $\mathrm{D}^{0}$ mesons in $\mathrm{Au}+\mathrm{Au}$ collisions at $\sqrt{s_{\mathrm{NN}}}=200 \mathrm{GeV}$ [12]. The data are compared to different energy loss model calcualtions. Right panel: $R_{\mathrm{AA}}$ of prompt $\mathrm{D}$ mesons and non-prompt $\mathrm{J} / \psi$ versus the number of participants [13].

strong suppression at $p_{\mathrm{T}}>3 \mathrm{GeV} / c$.

The ALICE experiment has measured the transverse momentum dependence of the nuclear modification factor $R_{\mathrm{AA}}$ for prompt $\mathrm{D}^{0}, \mathrm{D}^{+}, \mathrm{D}^{*+}$ and $\mathrm{D}_{\mathrm{s}}^{+}$mesons at mid-rapidity in the $7 \%$ most central lead-lead collisions at $\sqrt{s_{\mathrm{NN}}}=2.76 \mathrm{TeV}[13,14]$. All D mesons show a strong suppression of their yield (factor of 4-5 at around $10 \mathrm{GeV} / \mathrm{c}$ ) and follow a similar trend at low $p_{\mathrm{T}}$, except the $\mathrm{D}_{\mathrm{s}}^{+}$ mesons, which are less suppressed. The $\mathrm{D}_{\mathrm{s}}^{+}$is of particular interest since it contains a charm and anti-strange quark. A higher production yield for $\mathrm{D}_{\mathrm{s}}^{+}$mesons at intermediate $p_{\mathrm{T}}$ is expected due to the enhancement of strangeness production and if it hadronises via recombination in the medium.

The centrality dependence of high- $p_{\mathrm{T}}$ prompt D mesons measured by ALICE $[13,14]$ and J/ $\Psi$ mesons from B decays measured by CMS [15] are shown in Fig. 6 (right panel). The D and B mesons were selected in a compatible $p_{\mathrm{T}}$ range, namely $\left\langle p_{\mathrm{T}}^{\mathrm{B}}\right\rangle \approx 11 \mathrm{GeV} / c$ and $\left\langle p_{\mathrm{T}}^{\mathrm{D}}\right\rangle \approx$ $10 \mathrm{GeV} / c$. The data indicate a larger suppression of D mesons in particular for the most central collisions. This is the first clear indication that in-medium parton energy loss decrease with increasing quark mass. However, further theoretical developments are needed to directly link these measurements to the drag diffusion coefficient of heavy quarks. This quantity was recently calculated in Lattice QCD [16].

To quantitatively understand the heavy ion data in terms of energy loss, it is important to disentangle hot nuclear matter effects from initial-state effects due to cold nuclear matter, such as the modification of the parton distribution functions in the nucleus [17] and saturation effects in the heavy flavour sector [18]. 

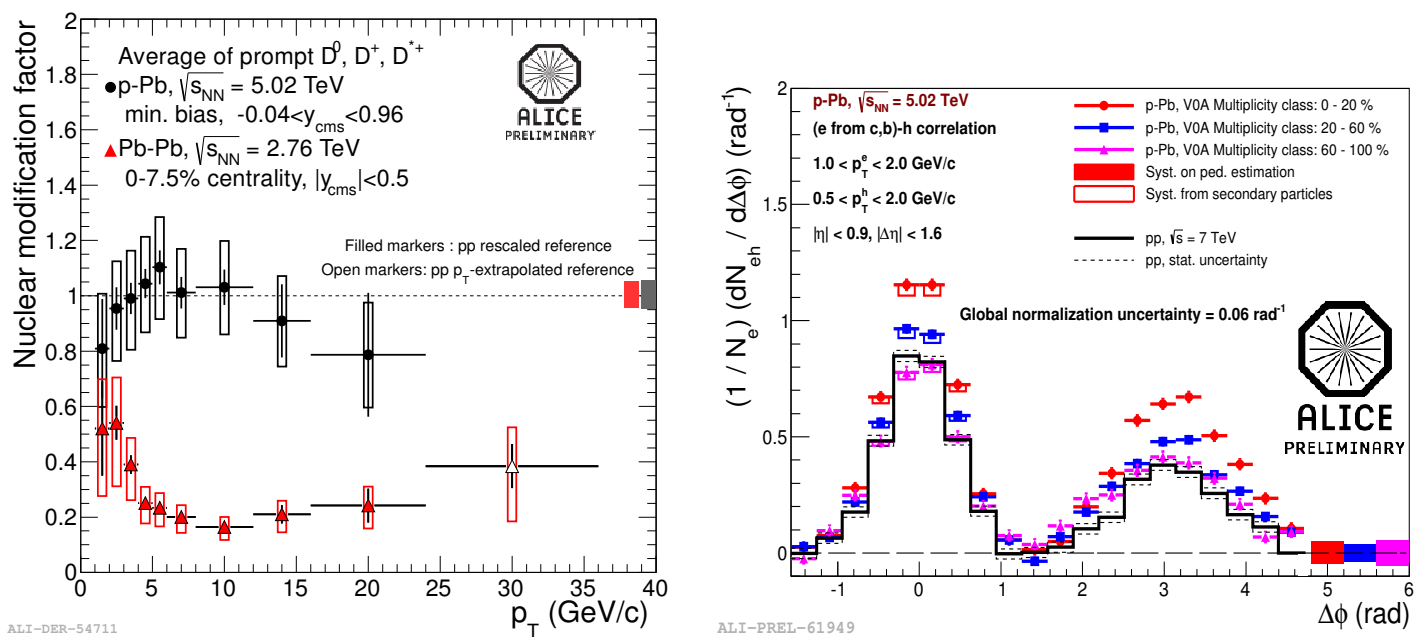

Figure 7: Left panel: $R_{\mathrm{AA}}$ of prompt $\mathrm{D}$ mesons (averaged) versus $p_{\mathrm{T}}$ for the $7.5 \%$ most central $\mathrm{Pb}-\mathrm{Pb}$ collisions (red triangle) at $\sqrt{s_{\mathrm{NN}}}=2.76 \mathrm{TeV}[13,14]$ and minimum bias p-Pb collisions at $\sqrt{s_{\mathrm{NN}}}=5.02 \mathrm{TeV}$ (black squares) [19]. Right panel: Azimuthal angular distribution between heavy flavour decay electrons and charged hadrons for three different multiplicity classes in p-Pb collisions at $\sqrt{s_{\mathrm{NN}}}=5.02 \mathrm{TeV}$ [20].

Initial-state effects can be investigated by measuring $\mathrm{D}$ production in $\mathrm{p}-\mathrm{Pb}$ collisions. The nuclear modification factor of the averaged prompt $\mathrm{D}^{0}, \mathrm{D}^{+}$and $\mathrm{D}^{*+}$ mesons in minimum bias $\mathrm{p}-\mathrm{Pb}$ at $\sqrt{s_{\mathrm{NN}}}=5.023 \mathrm{TeV}$ is depicted in Fig. 7 (left panel). The $R_{\mathrm{AA}}$ is compatible with unity within systematic uncertainties over the full $p_{\mathrm{T}}$ range. The data are compared with $\mathrm{pQCD}$ calculations based on the HVQMNR model calculations [3] combined with the EPS09 modifications of the parton distribution functions [17] and with a Color Glass Condensate-based calculation [18]. Both theoretical models describe the data within experimental uncertainties. Thus, the strong suppression of the heavy flavour hadron yield observed in central $\mathrm{Pb}-\mathrm{Pb}$ collisions is indeed a final-state effect, arising from the hot QCD matter.

The right panel of Fig. 7 depicts the azimuthal angular distribution between heavy flavour decay electrons and charged hadrons for three different multiplicity classes in $\mathrm{p}-\mathrm{Pb}$ collisions at $\sqrt{s_{\mathrm{NN}}}=$
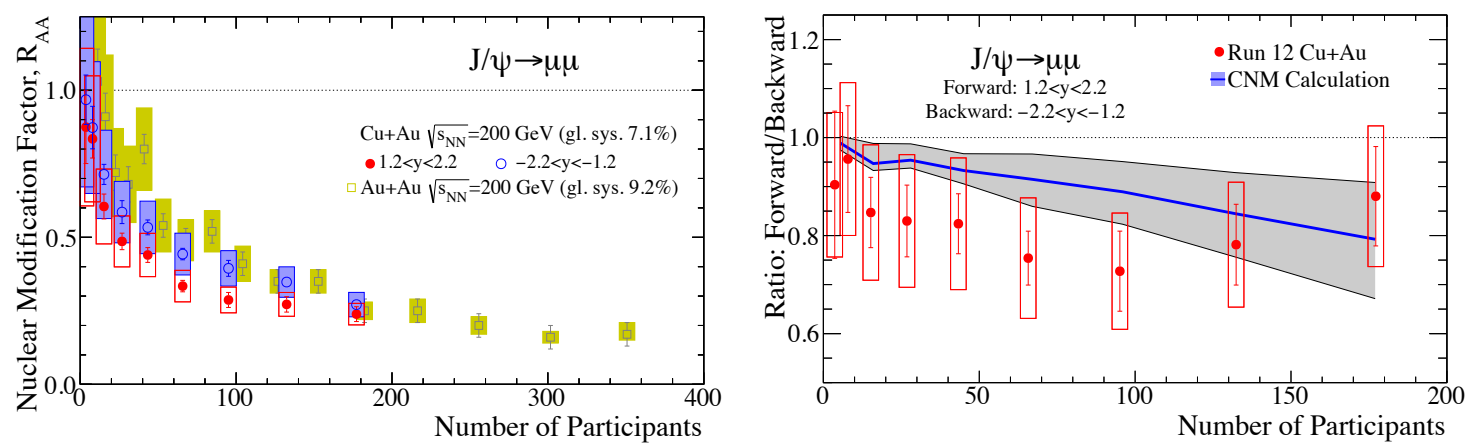

Figure 8: Left panel: Centrality dependence of the $J / \psi$ production in forward rapidity in $\mathrm{Cu}+\mathrm{Au}$ and $\mathrm{Au}+\mathrm{Au}$ collisions at $\sqrt{s_{\mathrm{NN}}}=200 \mathrm{GeV}$ (in terms of the number of participants) [27]. Right panel: Forward-backward ratio of the $J / \psi$ production yield in $200 \mathrm{GeV} \mathrm{Cu}+\mathrm{Au}$ collisions. [28]. 


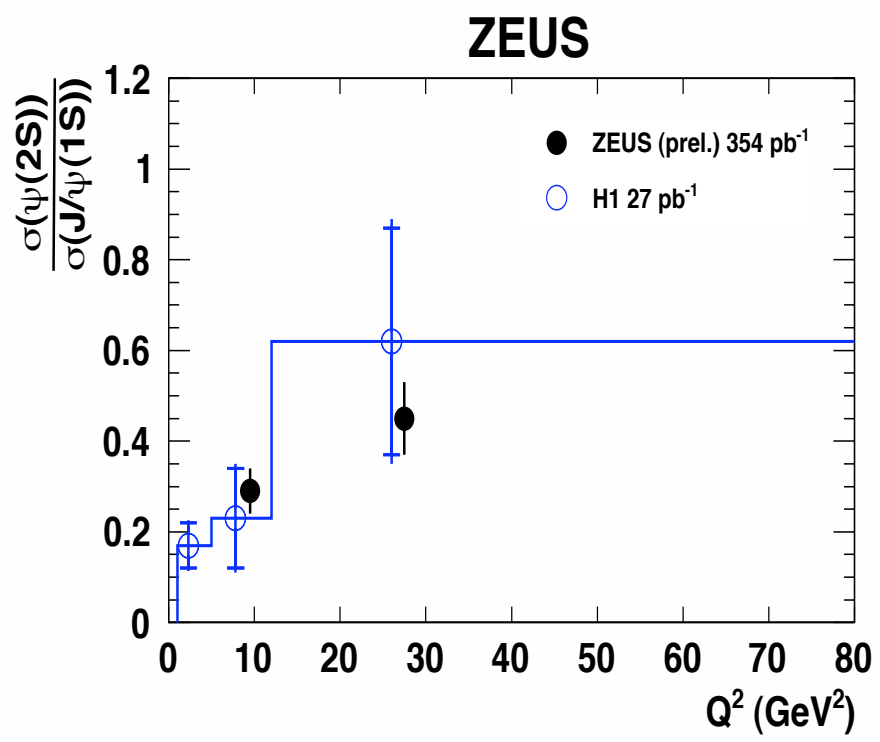

Figure 9: Exclusive production of $\psi(2 S)$ and $J / \psi(1 S)$ in electron-hadron deep-inelastic scatterings, measured by the H1 and ZEUS experiments [29].

$5.02 \mathrm{TeV}$ [20], measured by the ALICE experiment. It was shown that the correlation yield for low- $p_{\mathrm{T}}$ electrons $(1-2 \mathrm{GeV} / c)$ exhibits a long-range correlation in $\eta$ direction for the highest multiplicity class $(0-20 \%)$ compared to the low multiplicity class $(60-100 \%)$ and elementary $p p$ interactions. This "double ridge-like structure" can be interpreted in terms of hydrodynamic expansion of the systems or with the Colour Glass Model [18].

The dissociation of quarkonium states (hidden charm and beauty) due to colour screening in the QGP is one of the "classic signatures" of deconfinement [21]. A sequential suppression of the quarkonium states, such as $\Upsilon(1 \mathrm{~S}), \Upsilon(2 \mathrm{~S})$ and $\Upsilon(3 \mathrm{~S})$, depends on their binding energy and the temperature of the surrounding medium, thus providing a so-called "QCD thermometer" [22]. However, it has been shown that yield enhancement via subsequent regeneration in QGP or at chemical freeze-out of quarkonium states due to the large heavy-quark multiplicity might play an important role at LHC energies $[23,24,25,26]$. Furthermore feed-down from higher quarkonium states has to be considered.

The centrality dependence of $J / \psi$ production in forward rapidity in $\mathrm{Cu}+\mathrm{Au}$ and $\mathrm{Au}+\mathrm{Au}$ collisions at $\sqrt{s_{\mathrm{NN}}}=200 \mathrm{GeV}$ is shown in the left panel of Fig. 8 [27]. The $R_{\mathrm{AA}}$ is stronger suppressed in $\mathrm{Cu}+\mathrm{Au}$ collision ( $\mathrm{Cu}$-going direction) compared to the $\mathrm{Au}+\mathrm{Au}$ data at the same number of participants. Furthermore, the forward-backward ratio of the $J / \psi$ production yield in $200 \mathrm{GeV}$ $\mathrm{Cu}+\mathrm{Au}$ collisions is well described by theoretical model calculations considering shadowing effects [28] (cf. Fig. 8, right panel).

The H1 and ZEUS experiments presented the exclusive production of $\psi(2 S)$ and $J / \psi(1 S)$ in electron-hadron deep-inelastic scattering (cf. Fig. 9). It was shown that the cross-section ratios is reasonably independent of $t, W, Q^{2}$. These data provide stringent constraints on theoretical model calculations. 

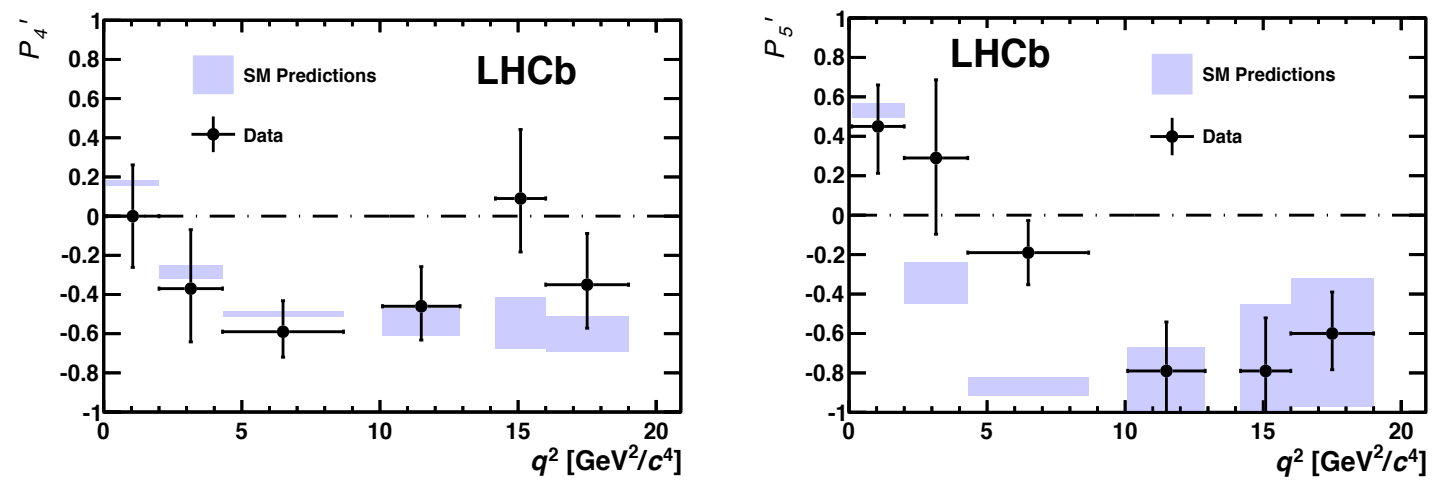

Figure 10: Form-factor-independent angular observables $P 4^{\prime}$ (left panel) and $P 5^{\prime}$ (right panel) measured in the decay $B^{0} \rightarrow K^{*}(892)^{0} \mu^{+} \mu^{-}$by the LHCb experiment in pp collisions at a centre-of-mass energy of 7 $\mathrm{TeV}$ [30]. The purple squares illustrate the Standard Model predictions.

\section{Rare decays, Mixing and New Physics searches}

Rare B decays are sensitive to New Physics beyond the Standard Model. Transitions of $b \rightarrow s$ only occur through loop/box processes where flavour is violated. The LHCb experiment observed a $3.7 \sigma$ discrepancy in the form-factor-independent angular observable $P 5^{\prime}$ parameter in the decay $B^{0} \rightarrow K^{*}(892)^{0} \mu^{+} \mu^{-}$in pp collisions at a centre-of-mass energy of $7 \mathrm{TeV}$ (integrated luminosity of $1.0 \mathrm{fb}^{-1}$ ) [30], This is not observed for $P 4^{\prime}$, as illustrated in Fig. 10 This discrepancy might be due to large power corrections or New Physics. LHCb has a factor of 3 more data on tape to examine these findings.

\section{Spectroscopy and Quarkonia}

In the area of Quarkonia-like states exotic hadrons consisting of four-quark states raised considerable attention. The charmonium(-like) states X(3872) was observed in more than one decay channel and the $\mathrm{Z}(4430)^{+}$was recently confirmed by LHCb. The Belle experiment found yet another charged $\mathrm{Z}$ in $B \rightarrow J / \psi K \pi$ channel. Similar studies are done for bottomonium(-like) states. Bfactories re-defined our understanding of hadrons and the classification is not possible within traditional quark model. Since the product branching fractions of the $X Y Z$ states are small $\left(\approx 10^{-6}\right)$, high luminosity experiments such as BESIII, LHCb and Belle II needed.

\section{Summary}

At this conference new data from the accelerator facilities DESY-HERA, BNL-RHIC, Tevatron, CERN-LHC and the B-factories and new theoretical developments have been presented. It has been shown that high precision measurements are needed to further constraint model calculations. Many more exciting results are expected from LHC Run2 (mid 2015) and after the LHC detector upgrades (2018) as well as from the future electron-hadron collider(s). 


\section{Acknowledgments}

I thank Antoni Szczurek, Ivan Belyaev, Martin Gorbahn, Anze Zupanc, Matthew Wing, Mykhailo Lisovyi, Aneta Iordanova, Antonio Manceau, Alessandro Grelli for fruitful discussions and for providing the data.

The European Research Council has provided financial support under the European Community's Seventh Framework Programme (FP7/2007-2013) / ERC grant agreement no 210223. This work was also supported by a Vidi grant from the Netherlands Organisation for Scientific Research (project number 680-47-232) and Projectruimte grants from the Dutch Foundation for Fundamental Research (project numbers: 10PR2884 and 12PR3083).

\section{References}

[1] B. Abelev et al. (ALICE Collaboration), JHEP 07, 191 (2012).

[2] B. Abelev et al. (ALICE Collaboration), JHEP 01, 128 (2012).

[3] M. Mangano, P. Nason and G. Ridolfi, Nucl. Phys. B 373, 295 (1992).

[4] M. Lisovyi for the H1 and ZEUS Collaborations, these proceedings.

[5] N. Zakharchuk for the ZEUS Collaboration, these proceedings.

[6] A. Grelli for the ALICE Collaboration, these proceedings.

[7] G. Aad et al. (ATLAS Collaboration), Phys. Rev. D 85, 052005 (2012).

[8] T. Sjöstrand and M. van Zijl, Phys. Lett., B 188, 149 (1987).

[9] R. Maciula and A. Szczurek, Phys. Rev. D87, 094022 (2013).

[10] A. van Hameren, R. Maciula and A. Szczurek, arXiv:1402.6972.

[11] A. Szczurek, these proceedings.

[12] L. Adamczyk et al. (STAR Collaboration), submitted to Phys. Rev Lett. (arXiv:1404.6185).

[13] A. Grelli, these proceedings.

[14] Suppression of high transverse momentum $\mathrm{D}$ mesons in central $\mathrm{Pb}-\mathrm{Pb}$ collisions at $\sqrt{s_{\mathrm{NN}}}=2.76 \mathrm{TeV}$, B. Abelev for the ALICE Collaboration, J. High Energy Phys. 9, 112 (2012).

[15] CMS Collaboration, CMSPASHIN12014.

[16] D. Banerjee, S. Datta, R. Gavai and P. Majumdar, Phys. Rev. D 85, 014510 (2012).

[17] K. J. Eskola H. Paukkunen and C. A. Salgado, JHEP 0904, 065 (2009).

[18] H. Fujii and K. Watanabe, Nucl Phys. A 920, 78 (2013).

[19] B. Abelev for the ALICE Collaboration, submitted to Phys. Rev. Lett. (arXiv:1405.3452), May 2014.

[20] E. Pereira de Oliveira Filho for the ALICE Collaboration, arXiv:1404.3983.

[21] T. Matsui and H. Satz, Phys. Lett. B 178, 416 (1986).

[22] S. Digal, P. Petreczky and H. Satz, Phys. Rev. D 64, 0940150 (2001).

[23] A. Andronic, P. Braun-Munzinger, K. Redlich and J. Stachel, Phys. Lett. B 571, 36 (2003). 
[24] P. Braun-Munzinger, J. Stachel, Phys. Lett. B 490, 196 (2000).

[25] Y. Liu, Z. Qu, N. Xu, P. Zhuang, Phys. Lett. B 678, 7 (2009).

[26] X. Zhao, R. Rapp, Nucl. Phys. A 859, 114 (2011).

[27] A. Iordanova, these proceedings.

[28] C. Aidala et al. (PHENIX Collaboration), submitted to Phys. Rev. C (arXiv:1404.1873).

[29] N. Kovalchuk, these proceedings.

[30] R. Aaij et al. (LHCb Collaboration), Phys. Rev. Lett. 111, 191801 (2013). 